\title{
INCIDENCIA DE UN PRESUPUESTO EN SEGURIDAD Y SALUD OCUPACIONAL EN LOS COSTOS Y GASTOS
}

\author{
INCIDENCE OF A BUDGET IN OCCUPATIONAL SAFETY AND HEALTH IN THE \\ COSTS AND EXPENSES
}

Cecina Mirtha Flores Pariona*

Unidad de Posgrado de la Facultad de Ciencias Contables

Universidad Nacional Mayor de San Marcos-UNMSM / Lima-Perú

[Recepción: Abril 2016/ Conformidad: Mayo 2016]

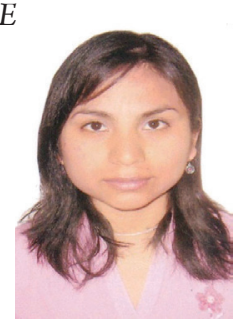

\section{RESUMEN}

Este artículo aborda aquellos desembolsos en seguridad y salud ocupacional que deben ser considerados como una inversión frente a todos aquellos costos y gastos que puedan desencadenarse, producto de un accidente o enfermedad ocupacional.

Un accidente deviene en costos de la mano de obra directa del accidentado, del compañero que emplea su tiempo para auxiliarlo, el personal de reemplazo, el costo de la mano de obra indirecta del supervisor y gastos como las indemnizaciones al accidentado, gastos médicos, hospitalización, multas, reparación de maquinarias y otros, que generan pérdidas para la empresa e incluso pueden afectar la productividad del trabajador, por las paralizaciones que ocasionan los accidentes.

Los empleadores tienen una responsabilidad moral y legal. Existe la ley de seguridad y salud en el trabajo, pero no siempre todos logran cumplirla, si bien es cierto en dicha ley no se menciona el uso de un presupuesto, es necesario que se cuente con esta herramienta de gestión y se realicen inversiones como dotación de equipos de protección personal, capacitaciones en seguridad, coberturas en salud entre otros. Para ello debe existir un compromiso tanto del empleador como el trabajador, ambos deben generar una cultura preventiva.

\section{Palabras Clave:}

Presupuesto, inversión, seguridad y salud ocupacional, costos, gastos, ley de seguridad y salud en el trabajo, productividad, empleador, trabajador y empresa.

\begin{abstract}
This article addresses those disbursements in occupational health and safety that should be considered as an investment facing all those costs and expenses that can be triggered, as a result of an accident or occupational illness.

An accident results in costs for the direct labor of the injured person, the partner who employs his time to assist him, the replacement personnel, the cost of the supervisor's indirect labor and expenses such as compensation for the accident, medical expenses, hospitalization, fines, repair of machinery and others. These generate losses for the company and can even affect the productivity of the worker, due to the paralyzes caused by the accidents.

Employers have a moral and legal responsibility. There is a law on health and safety at work, nevertheless not always everyone is able to follow it, although it is true that this law does not mention the use of a budget, it is essential to have this management tool and investments that are made as provision of personal protection equipment, safety training, health coverage, among others. For this, there must be a commitment of both employer and worker, they all must generate a preventive culture.
\end{abstract}

\section{Keywords:}

Budget, investment, occupational safety and health, costs, expenses, occupational safety and health law, productivity, employer, worker and company.

\footnotetext{
*Egresada de la Maestría en Contabilidad con mención en Costos y Presupuestos en la Gestión Estratégica - UNMSM CPC-UNAC.Email.Cecifp19@gmail.com
} 


\section{INTRODUCCIÓN}

Mayormente las empresas en el Perú no tienen una cultura de inversión en seguridad y salud ocupacional; no cuentan con un presupuesto definido para este concepto; sin embargo, esto tiene un impacto en el costo de producción, dado que si el trabajador no se encuentra seguro en el ambiente de trabajo no se comprometerá en la realización de su trabajo por temores inherentes de poder salir dañado en el proceso de producción, su rendimiento se verá disminuido y esto se traduce en una baja rentabilidad para la empresa.

La implementación efectiva de una inversión nos asegura una producción sin paralizaciones, con menores costos y un ambiente adecuado de trabajo. Una prevención cuesta menos que una corrección, los costos y gastos de un accidente se pueden elevar si consideramos las horas hombre perdidas, la baja de rendimiento de las personas, los pagos de seguros e indemnizaciones, las multas impuestas y hasta el deterioro de la imagen empresarial. Por ello, lo que todo empresario debe aspirar es a mantener a los empleados sanos, tanto a nivel físico como mental, brindando un ambiente de satisfacción incrementando la productividad.

Contar con un presupuesto como una herramienta de gestión en la inversión de seguridad y salud ocupacional, muestra el interés de minimizar las pérdidas mediante una gestión eficiente con la finalidad de prevenir todo tipo de accidentes y enfermedades y contribuyendo al logro de los objetivos de la empresa.

\section{MARCO TEÓRICO}

La importancia de presupuestar en materia de seguridady salud en el trabajo es una responsabilidad de la Gerencia, buscar reducir los riesgos laborales y poder garantizar la integridad de los trabajadores, brindándoles las condiciones para un buen desempeño, eficiencia y eficacia mediante el trabajo seguro.

El papel importante que juega el Gerente es reducir los riesgos, dado que existe una suma de costos y gastos como son las paralizaciones que produciría un accidente de trabajo, el costo de la mano de obra directa, personal accidentado; personal de reemplazo; el costo de la mano de obra indirecta, como es del supervisor que debe monitorear las causas del accidente; el costo de la mano de obra de los compañeros de trabajo que auxilian al accidentado; entre otros. Con respecto a los gastos, se pueden considerar los gastos médicos, las posibles indemnizaciones, multas $y$, algunas veces, esto puede conllevar al cierre temporal del negocio.

Un personal que se siente protegido es más rentable de aquel que se ve desprotegido y aislado. Dado que es de fácil percepción por los trabajadores, identificar si la compañía para la cual trabajan hace una inversión en seguridad y salud ocupacional. Son ellos los testigos de que su empleador tiene una preocupación por el bienestar de sus trabajadores.

El personal representa un recurso muy importante, se puede tener la mejor tecnología, dotarse de equipos y herramientas sofisticadas, pero descuidar al personal provocaría la reducción de su productividad.

Preocuparse por los colaboradores agrega valor a la empresa. El empresario debe ser preventivo antes que correctivo; dado que conlleva a un menor gasto.

No solo el empresario es responsable de la seguridad en el trabajo sino que todos los miembros que integren la empresa.

En nuestro país es notorio el compromiso que tiene las mineras con respecto al tema de seguridad y salud en el trabajo, por el gran impacto que tiene este tipo de sector $y$ porque son monitoreados constantemente; A pesar de ello, no se debe dejar de lado los otros sectores, dado que toda empresa cuenta con un activo importante como es el personal.

Inicialmente se ponía mucho énfasis al cumplimiento de leyes y normas acerca de salud y seguridad ocupacional, por parte de las empresas mineras realizando supervisiones a las mismas; sin embargo, hoy en día en Perú, su aplicación se ha extendido a través de una ley en Seguridad y Salud en el Trabajo que debería ser aplicado por todas las empresas; pero a la fecha no es así, como sabemos la creación de normas no quiere decir que automáticamente todos se acojan a ella, por eso, la importancia de este trabajo de investigación, radica en que no es válido estar acorde con la ley para evitar las multas, sino que el impacto es mayor con respecto a la productividad del trabajador, trayendo mejores resultados para le empresa. 
El destinar un presupuesto de seguridad y salud ocupacional tiene un fuerte impacto sobre los comportamientos de los miembros de la empresa, mostrará individuos activos y eficientes a diferencia de un ambiente sin protección, será extremadamente difícil organizarse y coordinar. Lo importante es la percepción del trabajador en su ambiente de trabajo.

Son los altos directivos quienes deben situar al presupuesto dentro de la reflexión estratégica.

El proceso de presupuesto es interactivo y nadie puede ser ajeno a ella, está ligado al seguimiento, análisis y el control. La elaboración del presupuesto busca controlar los gastos, pretende minimizar los riesgos a futuro, reconduce a alcanzar los objetivos trazados. El presupuesto expresa en forma monetaria los objetivos a conseguir.

Asimismo, un gerente está en la obligación de hacer un análisis reflexivo, ver donde se encuentra su empresa y a donde apunta llegar, y en base a ello, plantear que se debe de hacer. La planeación reduce las incertidumbres que puedan acontecerse, anticiparse a los cambios mediante la asignación de un presupuesto, contemple esto.

Toda empresa necesita de recursos para permanecer en el tiempo y poder alcanzar sus objetivos organizacionales y uno de los recursos en que se debe invertir, es en la protección del recurso humano, a través de un presupuesto en seguridad y salud ocupacional. Dado que el recurso humano es trascendental, porque de éste dependen los otros recursos.

Los accidentes constituyen un fuerte golpe en una empresa, porque deviene en ausencia de personal en sus puestos de trabajo, la productividad es baja debido al atraso de los productos o servicios, la imagen se ve mermada y la motivación del personal disminuye, producto de todo lo que sucedió en su entorno.

Por medio de un plan en seguridad y salud ocupacional se busca minimizar los riesgos y reducir costos operativos, incrementar la productividad y maximizar la rentabilidad, a través de un trabajador motivado.

La administración tiene un rol importante, porque es quien previene y controla las causas de pérdidas por accidentes. Por lo que es importante el desarrollo de capacitaciones al personal en materia de seguridad. Demostrando de este modo, que el recurso humano está involucrado en los casos de accidentes, como de los incidentes.

\section{RESULTADOS}

La gerencia realizó pequeñas inversiones en materia de seguridad y salud ocupacional; si bien es cierto, no asignan un presupuesto para este concepto en específico, sí hay una constante preocupación por alinearse a la Ley de Seguridad y Salud en el Trabajo N²9783-2011-TR.

En el Perú se conoce que abunda la informalidad, también están las empresas que siendo formales, como es el caso de la empresa estudiada, no llegan a cumplir con todas las exigencias en seguridad y salud en las actividades laborales y solo toman algunas acciones, cuando son observados por el Ministerio de Trabajo.

Uno de los principales pasos que debe seguir la empresa es establecer una adecuada política de seguridad y salud en el trabajo, en la cual se evidencie el compromiso de la alta dirección, en la toma medidas preventivas para minimizar la probabilidad de que ocurran accidentes y/o enfermedades ocupacionales.

\section{Principales riesgos identificados por procesos}

- Proceso de corte, el personal en este proceso está expuesto a una serie de riesgos por las diferentes actividades que realiza desde el traslado de la tela, uso de moldes filosos, preparación de la máquina troqueladora y corte de la tela. A pesar de que, estas actividades califican como rutinarias, ello no implica que el riesgo de accidentes sea menor.

El personal desde que realiza la manipulación de las cargas puede lesionarse la espalda e incluso cortarse con los troqueles filosos o sufrir atrapamiento de manos por la máquina troqueladora.

- Proceso de aparado, este proceso tiene tres actividades puntuales como la costura, remallado y colocación de ojalillos.

En todo este proceso están expuestos a punciones con las agujas tanto de la máquina de coser y rema- 
lladora. Con respecto a la máquina de hacer ojalillos pueden sufrir aplastamiento de dedos.

Además, el personal hace uso de herramientas manuales como tijeras y piqueteras, que en cierto nivel es un riesgo menor con respecto al proceso anterior.

- Proceso de Inyectado, la empresa cuenta con máquinas estacionarias y rotativas, ambas pueden ocasionar atrapamiento y cortes en las manos. Incluso, las rotativas pueden ocasionar golpes debido a que el soporte está en movimiento.

Ambas máquinas inyectan el PVC (policloruro de vinilo), al realizar esta operación, las superficies se mantienen calientes pudiendo ocasionar leves quemaduras.

- Proceso de Acabado, en este proceso los riesgos pueden considerarse leves, debido a que las tareas que se realizan en este proceso no implican el uso de maquinarias, como en otros procesos mencionados anteriormente.

En este proceso se utilizan herramientas manuales como cuchillas, tijeras y piqueteras que pueden conllevar a cortes.

Las actividades en este proceso se consideran rutinarias, y pueden desencadenar en dolencias por tratarse de movimientos repetitivos. Es decir, que se realizan tareas o actividades muchas veces en un corto periodo de tiempo.

\section{Efecto en la Productividad}

No contar con presupuesto en seguridad y salud ocupacional afecta a la productividad, dado que el rendimiento del trabajador no será el mismo, que en un escenario de accidente o en un ambiente de riesgos.

Todas las personas somos diferentes y no tenemos la misma capacidad de resistencia a los riesgos, todos procesamos la información de manera diferente.

A continuación detallamos el impacto ocasionado por los accidentes en términos de unidades y el importe en soles. (Ver Figura $\mathrm{N}^{\circ} 01$ )

- Las unidades dejadas de producir fueron de 93, 846, durante el periodo 2012 al 2015, esto representa en soles el importe de S/.488, 938.

- El mayor número de unidades dejadas de producir a causa de los accidentes, se dieron en el año 2015, como se muestra la Figura $N^{\circ} 01$, las unidades producidas con accidentes fueron de 236 , 283 y si la comparamos con las unidades que se hubieran producido sin ocurrencia de accidentes, las unidades producidas por trabajador hubieran ascendido a 260,352, esto ocasiona una perdida en unidades valorizadas de S/. 125,399 soles.

- En el 2014 existe una ligera disminución con respecto al año 2013; sin embargo, sigue existiendo pérdidas, que ascienden a $S / .119,642$ soles.

Es importante que se valorice el tiempo en el que se producen los accidentes porque eso impacta en la productividad del trabajador, las horas que se toman para auxiliar al accidentado, hacer seguimiento del evento por parte de supervisor, demandan un tiempo extra, la paralización de la producción debido a que el accidentado no continua con el proceso productivo porque no se minimizaron los riesgos o simplemente se designó poca inversión en materia de seguridad y salud ocupacional. 


\section{Figura No 01:}

Efectos en la productividad, Multas por parte de los inspectores

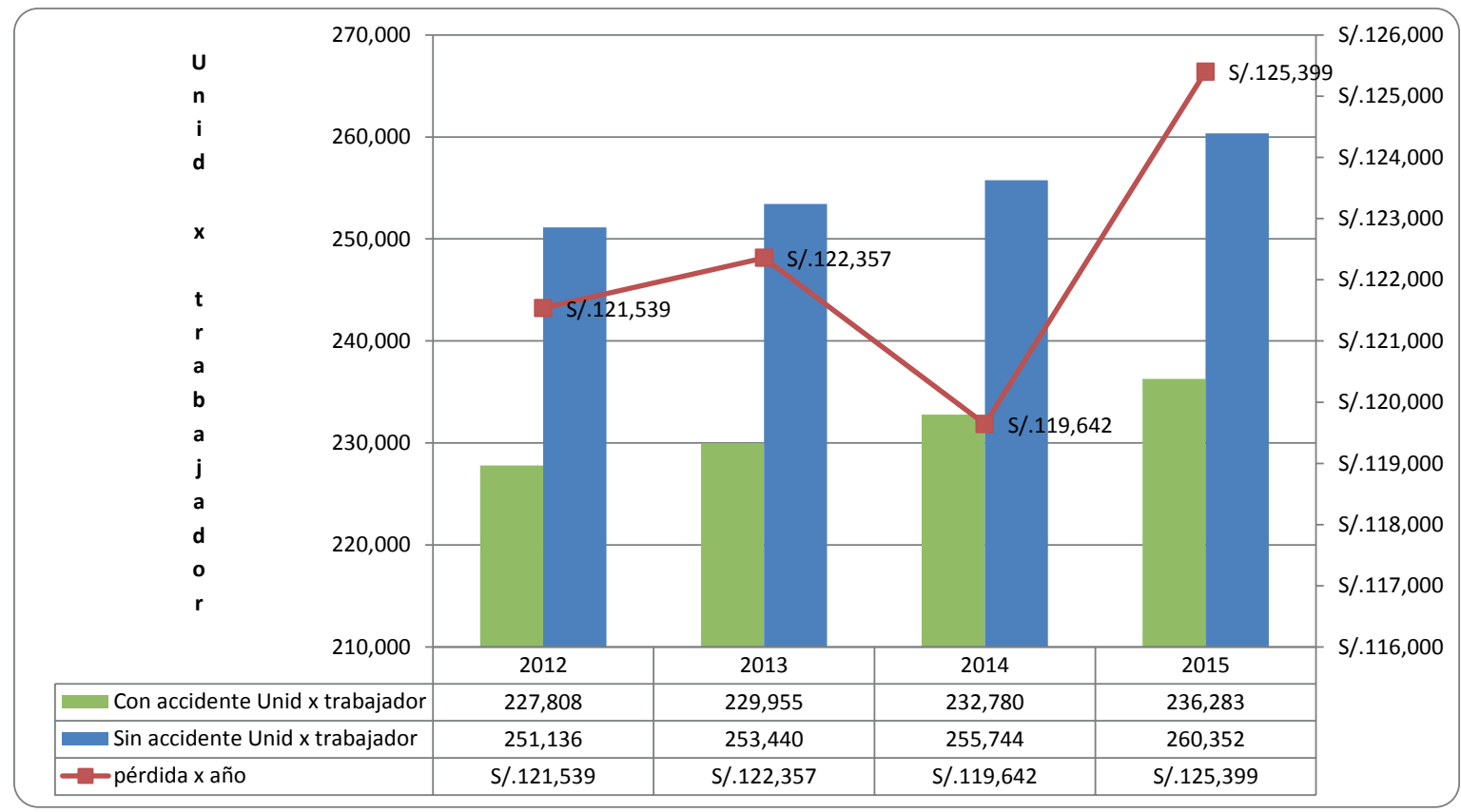

Fuente: Empresa de Zapatillas (2012-2015).Elaboración Propia.

\section{Gastos}

No contar con un presupuesto en seguridad y salud ocupacional conlleva a una serie de gastos como son:

- Indemnizar al trabajador afectado producto del accidente.

- Multas por parte de los inspectores del Ministerio de Trabajo, que están en base a un porcentaje de la unidad impositiva tributaria (UIT) que para el año 2016 asciende a S/.3,950.

- El costo de reparación de maquinaria italiana, considerando que las piezas no son tan fáciles de conseguir en el mercado peruano.

- Cubrir los gastos médicos y asistencia hospitalaria.

A continuación se muestra en la Figura $\mathrm{N}^{\circ} 02$, los desembolsos realizados por la empresa de zapatillas durante el período $2012 \mathrm{al} 2015$, el total de gasto en ese rango de tiempo asciende a $S / .14,785$, evidenciándose un mayor gasto en el año 2015 con respecto a los años anteriores, el importe registrado es de $S / 7,600$ representando un 51\% del total.
Figura No 02:

Gastos de la empresa de zapatillas

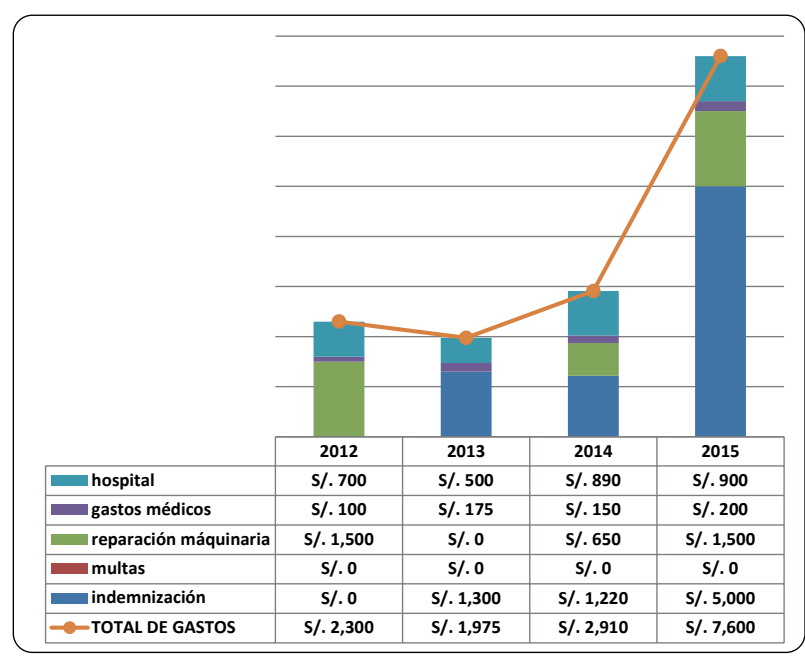

Fuente: Empresa de Zapatillas (2012-2015).Elaboración Propia.

Como se puede apreciar en la Figura $\mathrm{N}^{\circ} 02$, la empresa no ha tenido multas por parte del Ministerio de Trabajo y Promoción del Empleo, en el periodo 
analizado, pero sí ha tenido una fuerte indemnización producto de un accidente.

El gerente general, busca minimizar los accidentes y por consecuencia los gastos relacionados con este concepto se verán reducidos.

Los accidentes y enfermedades laborales afectan a la empresa, porque a mayores gastos se reduce la utilidad.

Los altos directivos de la empresa de zapatilla, tienen una constante preocupación de proporcionar un ambiente de trabajo agradable y están conscientes de que es indispensable asignar un presupuesto en seguridad y salud ocupacional, dado que constituye una inversión y no un gasto.

La empresa busca minimizar todos aquellos gastos que provienen de enfermedades o accidentes de trabajo y velan por el bienestar del trabajador para que la productividad no se vea afectada.

La gestión en seguridad y salud ocupacional consiste en el control de los riesgos potenciales que desencadenen la ocurrencia de accidentes o incidentes.

Dentro de la propuesta, también se incluye contar con una cobertura de seguro complementario de trabajo de riesgo y salud.

\section{CONCLUSIONES}

1. El objetivo fundamental de asignar un presupuesto en seguridad y salud ocupacional es contribuir al bienestar del trabajador, impactando positivamente en la productividad, y así, la empresa pueda maximizar sus utilidades; y no solo ello, sino cumplir con la ley de seguridad y salud en el trabajo, que por estos días no solo son obligatorias para las empresas mineras, sino que su alcance es para todas las organizaciones.

2. Con un presupuesto asignado la empresa reducirá los costos de mano de obra directa e indirecta, que involucra los accidentes o enfermedades ocupacionales y aquellos gastos que se derivan a consecuencia de ello.

3. No solo basta asignarles un presupuesto en seguridad y salud ocupacional, sino que se requiere el compromiso del personal y del empleador, para llevar a a cabo toda esta implementación.
4. Medir la gestión de las áreas a través del presupuesto, reducir los riesgos a los cuales están expuestos los trabajadores, sabiendo que toda actividad lleva consigo un riesgo inherente.

5. Entre los objetivos principales de contar con un presupuesto en seguridad y salud ocupacional, es prevenir todos aquellos acontecimientos que restan financieramente a la empresa, producto de los tiempos improductivos y aquellos costos asociados con enfermedades o accidentes ocupacionales, que algunas vez puedan afectar a la imagen de la empresa.

\section{REFERENCIAS BIBLIOGRÁFICAS}

1. CHINCHILLA, R. (2015). salud y seguridad en el trabajo (quinta ed.). costa rica: editorial universidad estatal a distancia.

2. DÍAZ, P. (2015). Prevención de Riesgos Laborales (Segunda ed.). Madrid, España: Ediciones Paraninfo, SA.

3. GARCÍA, J. (2014). Contabilidad de Costos (Cuarta ed.). México: Mc Graw-Hill / Interamericana Editores S.A.

4. Ley $N^{\circ} 29783$. (2011). Ley de Seguridad y Salud en el Trabajo.

5. RAMÍREZ, C. (2005). Seguridad Industrial. Un enfoque Integral (Segunda ed.). México: Editorial Limusa S.A. 